\title{
A prospective randomised trial to study the role of levamisole and interferon alfa in an adjuvant therapy with 5-FU for stage III colon
}

\section{cancer}

\section{W Schippinger ${ }^{*, 1}$, M Jagoditsch ${ }^{2}$, C Sorré2 ${ }^{2}$ M Gnant ${ }^{3}$, G Steger ${ }^{4}$, H Hausmaninger ${ }^{5}$, B Mlineritsch ${ }^{5}$, R Schaberl-Moser ${ }^{1}, \mathrm{HJ}$ Mischinger ${ }^{6}$, F Hofbauer ${ }^{7}$, P Holzberger ${ }^{8}$, M Mittlböck ${ }^{9}$ and $R$ Jakesz $^{3}$ for the Austrian Breast and Colorectal Cancer Study Group}

\begin{abstract}
'Medical Department, Division of Oncology, Graz Medical School, A-8036 Graz, Austria; ${ }^{2}$ St Veit Hospital, A-9300 St Veit/Glan, Austria; ${ }^{3}$ Department of Surgery, Vienna Medical School, A- 1090 Vienna, Austria; ${ }^{4}$ First Medical Department, Division of Oncology, Vienna Medical School, A- 1090 Vienna, Austria; ${ }_{5}^{5}$ Third Medical Department of the Private Medical Paracelsus University Salzburg, A-5020 Salzburg, Austria; ${ }^{6}$ Department of Surgery, Graz Medical School, A-8036 Graz, Austria; 'Department of Surgery, Oberpullendorf Hospital, A-7350 Oberpullendorf, Austria; ${ }^{8}$ Department of Surgery, Innsbruck Medical School, A-6020 Innsbruck, Austria; ${ }^{9}$ Core Unit for Medical Statistics and Informatics, Vienna Medical School, A- 1090 Vienna, Austria
\end{abstract}

The purpose of this trial was to examine the efficacy of the addition of levamisole (LEV) or interferon alfa (IFN) to an adjuvant chemotherapy with 5 -fluorouracil (5-FU) in patients with stage III colon cancer. According to a $2 \times 2$ factorial study design, 598 patients were randomly assigned to one of four adjuvant treatment arms. Patients in arm one received 5-FU weekly for I year, patients in arm two 5-FU plus LEV, in arm three 5-FU plus IFN and patients in arm four 5-FU, LEV and IFN. The relative risk of relapse and the relative risk of death were significantly higher for patients treated with LEV compared with those without LEV treatment (HR I.452, $95 \% \mathrm{Cl}$ I.135-1.856, $P=0.0028$; HR I.506, $95 \% \mathrm{Cl}$ I.I50-1.973, $P=0.0027$, respectively). No significant impact on survival was observed for therapy with IFN in the univariate analysis. The addition of LEV to adjuvant 5-FU significantly worsened the prognosis of patients with stage III colon cancer. Interferon alfa had no significant influence on survival when combined with adjuvant 5-FU, but increased the toxicity of therapy substantially.

British Journal of Cancer (2005) 92, I655- |662. doi:I0.1038/sj.bjc.6602555 www.bjcancer.com

Published online 26 April 2005

(c) 2005 Cancer Research UK

Keywords: colon cancer; adjuvant therapy; levamisole; interferon

Colorectal cancer is the second most common malignant disease in developed countries (Parkin et al, 2001). About $50 \%$ of patients with colorectal cancer who undergo surgical treatment subsequently develop recurrent disease ultimately leading to incurability. Adjuvant chemotherapy containing 5-fluorouracil (5-FU) after complete surgical removal of the primary tumour has been shown in randomised trials to decrease the risk of disease relapse (Wolmark et al, 1988; Laurie et al, 1989). Based on reports suggesting a possible benefit of combining 5-FU with levamisole (LEV), an anthelmintic drug with immunomodulatory activity, a large Intergroup trial was launched in 1984 comparing 5-FU plus LEV for 1 year with observation in patients with curatively resected stage III colon cancer. 5-Fluorouracil with LEV reduced the risk of cancer recurrence by $41 \%$ and the overall death rate by $33 \%$ (Moertel et al, 1990). In conclusion of these results, 5-FU and LEV were considered to be standard adjuvant treatment for stage III colon cancer for several years (NIH, 1990).

*Correspondence: Dr W Schippinger, Klinische Abteilung für Onkologie, Medizinische Universitätsklinik Graz, Auenbruggerplatz 15, A-8036 Graz, Austria; E-mail: walter.schippinger@klinikum-graz.at

Received 29 November 2004; revised 3 March 2005; accepted 4 March 2005; published online 26 April 2005
However, other authors failed to show a benefit by adding LEV to adjuvant 5-FU chemotherapy (Sertoli et al, 1987), and LEV as a single-agent therapy has been shown to be ineffective as adjuvant therapy (Arnaud et al, 1989). In addition, the combination of LEV with 5-FU in advanced colon cancer was shown not to be superior to 5-FU single-agent therapy (Buroker et al, 1985). At the time the present study was initiated, combination of LEV and 5-FU was considered to be standard therapy to eliminate minimal residual disease. Consequently, this trial was designed to evaluate this combination treatment in comparison with an experimental approach in patients with stage III colon cancer.

One way to enhance the antitumour activity of 5-FU was the implementation of interferon alfa (IFN) in treatment strategies of colorectal cancer. Interferon alfa as an immunomodulatory cytokine had been shown to increase the cytotoxic activity of 5-FU in human cancer cell lines (Wadler et al, 1990). Several clinical trials confirmed an increased responsiveness by addition of IFN to 5-FU in the treatment of metastatic colon cancer (Wadler et al, 1989, 1991). According to these promising data, the second aim of the present study was to examine the efficacy of IFN plus 5 -FU as adjuvant therapy for stage III colon cancer in a $2 \times 2$ factorial study design. 


\section{MATERIALS AND METHODS}

This multicentre trial was initiated by the Austrian Breast and Colorectal Cancer Study Group (ABCSG) and involved 41 participating hospitals. The protocol was approved by the ethics committees of the participating institutions. Enrolment of patients began in October 1991 and was completed in April 1999.

\section{Patient selection}

To be eligible, patients had to fulfil the following inclusion criteria: histologically proven carcinoma of the colon with lymph node metastasis (stage III tumour according to the International Union Against Cancer); potentially curative resection without gross or microscopic evidence of residual disease; age between 18 and 80 years; WHO performance status of 0 or 1 ; absence of severe concomitant disease and other malignancies; and adequate bone marrow function. The following were considered as exclusion criteria: prior or concomitant chemotherapy, immunotherapy and radiotherapy, carcinoma of the rectum defined as tumour below the anatomical rectosigmoidal border line or within $16 \mathrm{~cm}$ from the anal verge measured by a nonflexible rectoscope. Other exclusion criteria were the presence of metastasis or a time period of more than 42 days from surgery to start of adjuvant therapy.

Written informed consent was obtained from all patients participating in this trial.

Site visits were performed in regular intervals to check the original data concerning eligibility and to review the documented chemotherapy and follow-up data.

\section{Surgical treatment}

A tumour of the caecum, the ascending colon or the hepatic flexure was resected with a right hemicolectomy with resection of the right colic artery and the ileocolic artery. A tumour of the colon transversum was treated by resection of the appropriate part of the colon including the hepatic and splenic flexure. Carcinomas of the descending colon were resected by a left hemicolectomy including the left colic artery. Finally, sigmoid tumours were treated by a sigmoid resection including the inferior mesenteric blood vessels. The resection of at least seven regional lymph nodes was recommended.

\section{Randomisation and stratification procedures}

Patients were stratified by sex, age ( $\leqslant 65 v s>65-80$ years), tumour size (T1 vs T2 vs T3 vs T4), number of involved lymph nodes ( $\leqslant 3 v s>3$ lymph nodes) and tumour differentiation (G1/2 vs G3/4). Randomisation was performed by computer, assigning the patients according to a $2 \times 2$ factorial study design to one of four postoperative treatment arms: (1) 5-FU, (2) 5-FU plus LEV, (3) 5-FU plus IFN and (4) 5-FU plus LEV plus IFN according to the method of Pocock and Simon (1975), which marginally balances the following criteria: sex, age ( $\leqslant 65 v s>65-80$ years), tumour size (T1 vs T2 vs T3 vs T4), number of involved lymph nodes ( $\leqslant 3$ vs $>3$ lymph nodes), tumour differentiation (G1/2 vs $\mathrm{G} 3 / 4)$ and state.

\section{Schedules of chemotherapy administration}

Adjuvant chemotherapy was required by the trial protocol to be started within 42 days after tumour resection. In treatment arm 1 , adjuvant therapy consisted of $5-\mathrm{FU} 450 \mathrm{mg} \mathrm{m}^{-2}$ as an intravenous bolus given initially on five consecutive days and then once weekly, starting on day 28 for 48 weeks ( $=52$ weeks of therapy). Patients in treatment arm 2 received 5-FU as in arm 1 and additionally LEV $50 \mathrm{mg}$ orally three times a day for three consecutive days (days $1-3$ ) every 14 days. In arm 3, patients received 5-FU as in arm 1 and interferon alfa-2C (Berofor ${ }^{\circledR}$, Boehringer Ingelheim $\mathrm{GmbH}$, Germany) at a dose of $3.5 \times 10^{6} \mathrm{U}$ subcutaneously three times a week (Monday, Wednesday, Friday) for 52 weeks. Arm 4 consisted of 5-FU plus LEV in the same schedule as in arm 2 and of IFN $3.5 \times 10^{6} \mathrm{U}$ subcutaneously three times a week (Monday, Wednesday, Friday) for 52 weeks.

In all four treatment arms, dose modifications were required for haematologic or other severe toxicity. According to the protocol, the 5 -FU dosage was reduced by $25 \%$ when patients developed leucopenia $\left(3.0-3.5 \times 10^{9} 1^{-1}\right)$ and/or thrombocytopenia $\left(<100 \times 10^{9}\right.$ and $\left.>75.10^{9} 1^{-1}\right)$. The dose was reduced by $50 \%$, when thrombocyte counts decreased to $50 \times 10^{9}-75 \times 10^{9} 1^{-1}$. In case of more severe leucopenia $\left(<3.0 \times 10^{9} 1^{-1}\right)$ and/or thrombocytopenia $\left(<50 \times 10^{9} 1^{-1}\right)$, treatment was delayed for 1 week.

\section{Follow-up}

During the first year after randomisation (= treatment period), patients were evaluated every 3 months, during years $2-5$ every 6 months and thereafter, once yearly until year 10 after randomisation. A physical examination with evaluation of body weight and performance status, determination of blood count, serum liver function parameters, carcinoembryonic antigen (CEA) and tests for occult blood in stool, as well as chest radiography and ultrasonography of the liver were performed at each follow-up visit. Colonoscopy was required during the first 5 years every 6 months, then once a year.

\section{Statistical methods}

The trial was planned as $2 \times 2$ factorial design with four arms (LEV $v s$ no LEV and IFN $v s$ no IFN). It was designed to detect a difference in 5 -year survival rate for each factor of $12 \%(59-71 \%)$ with a power of $85 \%$ and a two-sided significance level of 0.05 , so that approximately 200 patients would be recruited for 3 years and followed-up for another 4 years or equivalently 225 observed deaths.

The intention-to-treat analysis included all eligible patients for whom complete baseline data were available. All patient data were collected at the study group's central data office and processed and analysed applying SAS software (SAS Institute, Cary/NC, USA). Distribution of prognostic factors to the treatment arms was described with frequencies for categorical variables and with medians for continuous data and tested with the $\chi^{2}$-test and Kruskal-Wallis test, respectively.

Overall survival (OS) was defined as the time between date of randomisation of primary colon cancer until date of last visit or date of death, independent of the cause of death. Disease-free survival (DFS) was defined as time from randomisation to either recurrence of colon cancer, occurrence of metastases, occurrence of a second primary cancer, or death without evidence of recurrence or a second primary tumour, or date of last visit.

Overall survival and DFS were estimated and graphically presented according to the method of Kaplan and Meier (1958). Differences between curves were assessed by the Mantel log-rank test for censored survival data (Mantel, 1966). The Cox proportional hazards model (Cox, 1972) was used to assess the prognostic values of treatment, sex, age, lymph node status, tumour grading, tumour stage, tumour localisation and number of surgically removed lymph nodes in univariate and multiple analyses and quantifies their effect by hazard ratios. Corresponding $95 \%$ confidence intervals are also given. Furthermore, it was applied to assess interactions between treatment and the other covariates and to detect nonproportionality. A Cox regression model with the time-dependent factor was modelled to assess the effect of the cumulative 5-FU dose, which increases with time from the first to the last treatment delivery. All $P$-values given are two-sided. 


\section{RESULTS}

\section{Patient characteristics}

A total of 598 patients entered this prospective randomised trial. A total of 22 erroneously randomised patients (3.7\%) were found to be ineligible and were therefore not included in the analysis. Four patients were removed from the statistical analysis for lack of any baseline or follow-up data. Statistical analyses were performed with the data of the remaining 572 eligible patients according to the intention-to-treat principle. Baseline patient and tumour characteristics are reported in Table 1 . The patient and tumour characteristics were well balanced between the four treatment arms. The median follow-up time for the study population was 84.3 months and $50 \%$ of the patients had a follow-up time of $65.2-108.2$ months.

\section{Treatment adherence and delivered 5-FU dose}

Adjuvant therapy as planned in the protocol was prematurely discontinued without evidence of tumour relapse in 25 patients (17.2\%) of the 5-FU single-agent group, in 29 patients $(20.7 \%)$ of the 5 -FU plus LEV group, in 52 patients $(36.6 \%)$ of the 5 -FU plus IFN group and in 72 patients $(49.7 \%)$ of the 5-FU/LEV/IFN triple-agent group. In most of the cases, the reasons for treatment discontinuation were toxicity and the patients' wish to discontinue therapy. The median, actually delivered 5-FU dose (5-FU dose given in grams per square metre) cumulated over time was $22.397 \mathrm{~g} \mathrm{~m}^{-2}$ in treatment arm $1,21.600 \mathrm{~g} \mathrm{~m}^{-2}$ in arm $2,17.118 \mathrm{~g} \mathrm{~m}^{-2}$ in therapy arm 3 and $13.500 \mathrm{~g} \mathrm{~m}^{-2}$ in arm 4 .

The median cumulatively delivered 5-FU dose was significantly lower in the therapy arms including LEV than in the treatment arms without LEV (17.953 vs $\left.20.828 \mathrm{~g} \mathrm{~m}^{-2}, P=0.0055\right)$ and also significantly lower in the therapy arms including IFN compared to the treatment arms without IFN $\left(15.647^{2}\right.$ vs $22.050 \mathrm{~g} \mathrm{~m}^{-2}$, $P<0.0001$ ).

As older patients may tend to have lower tolerability for treatment combinations, the association between age and cumulatively delivered 5 -FU dose ( $<80 v s \geqslant 80 \%$ of planned dose) was investigated in a cross-table. The results showed a trend to higher
5-FU dose for younger patients in treatment arms 1 and 2, where no IFN was applied. The two arms with IFN treatment (groups 3 and 4) showed a higher rate of patients with lower cumulatively delivered 5-FU dose; however, lower 5-FU dose seemed not to be related to age of patients.

\section{Tumour relapse and disease-free survival}

To date, 259 patients have relapsed. Tumour relapse was documented in 136 of 285 patients $(47.7 \%)$ in the therapy arms containing LEV (treatment arms 2 and 4), in 113 of 287 patients $(39.4 \%)$ in the therapy arms not containing LEV (arms 1 and 3), in 134 of 287 patients (46.7\%) treated with IFN (arms 3 and 4) and in 125 of 285 patients (43.8\%) not treated with IFN (arms 1 and 2).

In univariate analyses, the relative risk of relapse in the patient cohort treated with LEV (treatment arms 2 and 4) was significantly higher than in the patient group that had not received LEV (arms 1 and 3) (HR 1.452, 95\% CI 1.135-1.856, log-rank test: $P=0.0028$ ). There was no statistically significant difference in the relative risk of relapse between the patient group treated with IFN and the group without IFN treatment (HR 1.019, 95\% CI 0.798-1.300, logrank test: $P=0.8826$ ).

Consequently, DFS was significantly shorter for patients treated with LEV compared to patients who had not received LEV.

No significant difference in DFS was observed between patients treated with IFN and patients treated without IFN.

Kaplan-Meier curves for DFS are shown in Figures 1 and 3.

\section{Overall survival}

Altogether, 217 of 572 eligible patients have died: 126 of 285 patients $(44.2 \%)$ in the therapy arms containing LEV (treatment arms 2 and 4), 91 of 287 patients (31.7\%) in the therapy arms not containing LEV (arms 1 and 3), 114 of 287 patients (39.7\%) treated with IFN (arms 3 and 4) and 103 of 285 patients (36.1\%) not treated with IFN (arms 1 and 2).

Univariate analyses revealed that patients treated with LEV had a significantly higher relative risk of death compared to those

Table I Clinical and pathological characteristics by treatment arms

\begin{tabular}{|c|c|c|c|c|c|}
\hline & 5-FU (\%) & 5-FU+LEV (\%) & 5-FU+IFN (\%) & 5-FU+LEV+IFN (\%) & $P$-value \\
\hline Number of patients & 145 & 140 & 142 & 145 & \\
\hline Median age (years) & 63.0 & 62.6 & 62.9 & 63.2 & 0.7249 \\
\hline Male/female & $51.7 / 48.3$ & $50.7 / 49.3$ & $51.4 / 48.6$ & $50.3 / 49.7$ & 0.9953 \\
\hline T category & & & & & 0.9897 \\
\hline $\mathrm{Tl}$ & 2.1 & 0.7 & 1.4 & 0.7 & \\
\hline $\mathrm{T} 2$ & 8.3 & 7.9 & 9.2 & 9.7 & \\
\hline T3 & 75.2 & 75.0 & 73.2 & 73.8 & \\
\hline $\mathrm{T} 4$ & 14.5 & 16.4 & 16.2 & 15.8 & \\
\hline$N$ category & & & & & $0.999 \mid$ \\
\hline $\mathrm{NI}$ & 55.8 & 55.0 & 54.9 & 56.5 & \\
\hline N2 & 29.0 & 29.3 & 31.0 & 28.3 & \\
\hline N3 & 15.2 & 15.7 & 14.1 & 15.2 & \\
\hline Grading & & & & & 0.9616 \\
\hline G1 and $G 2$ & 67.6 & 69.1 & 70.4 & 69.7 & \\
\hline G3 and G4 & 32.4 & 30.9 & 29.6 & 30.3 & \\
\hline Location of tumour & & & & & 0.4467 \\
\hline Caecum and right colon & 30.3 & 32.9 & 25.4 & 29.0 & \\
\hline Left colon and sigmoid & 46.9 & 46.4 & 48.6 & 54.5 & \\
\hline Flexures and transverse colon & 22.8 & 20.7 & 26.1 & 16.5 & \\
\hline
\end{tabular}

5-FU = 5-fluorouracil; LEV = levamisole; IFN = interferon alfa. 


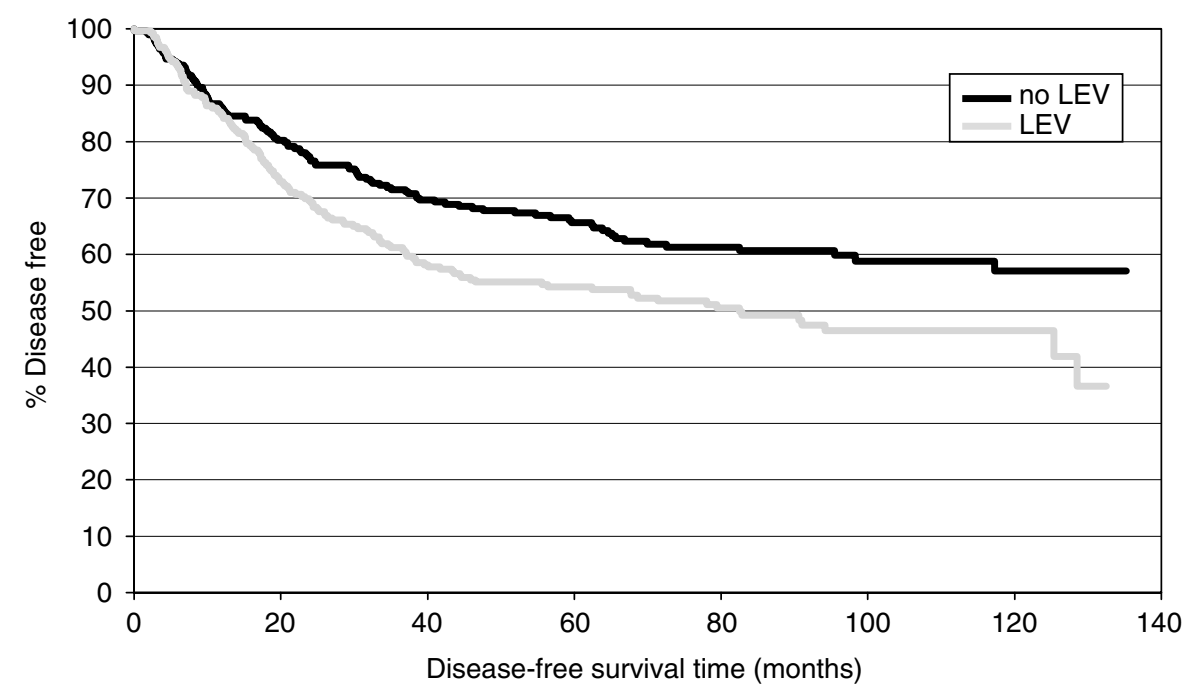

Figure I Disease-free survival: LEV vs no LEV.

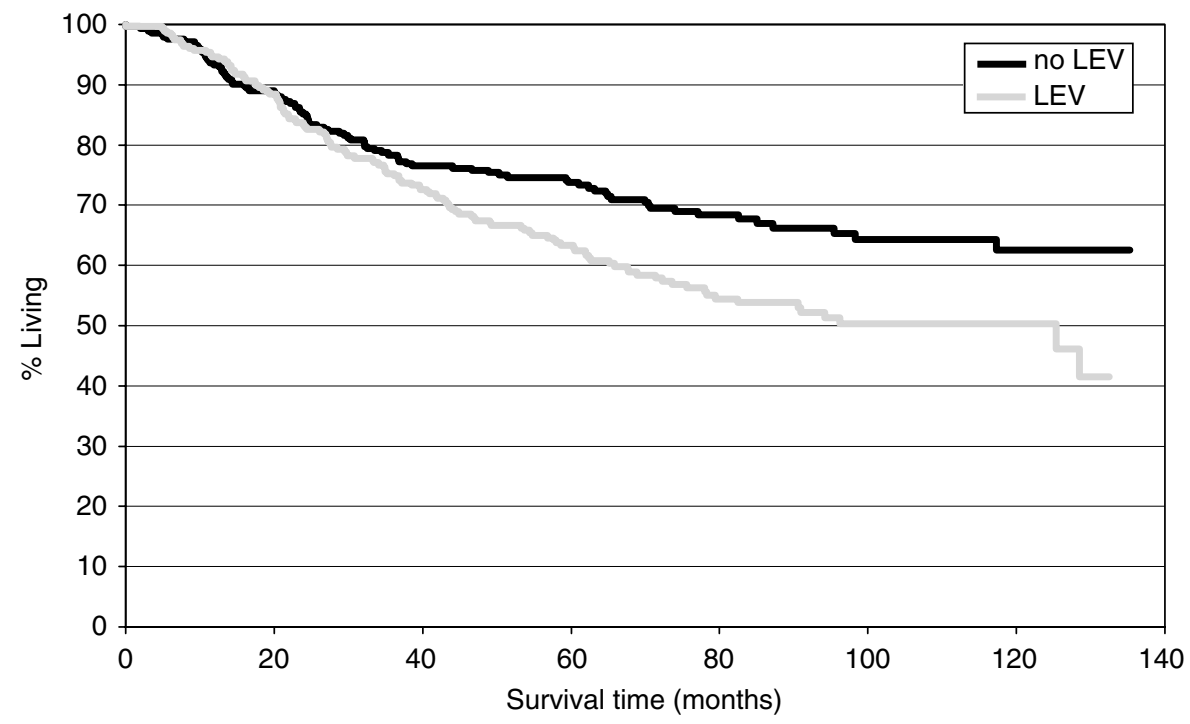

Figure 2 Overall survival: LEV vs no LEV.

patients who were not treated with LEV (HR 1.506, 95\% CI 1.150 1.973, $P=0.0027)$.

No significant difference in the relative risk of death was observed between the patient group treated with IFN and the group treated without IFN (HR 1.046, 95\% CI 0.802-1.366, $P=0.738$ ).

Considering these data, it follows that patients treated with LEV had a significant disadvantage in OS compared to those patients who did not receive LEV.

There was no significant difference in OS between patients treated with IFN and the patients without IFN therapy.

Kaplan-Meier curves for OS are presented in Figures 2 and 4.

\section{Multiple analyses of prognostic factors}

Cox regression analysis revealed age, sex, tumour size, the number of metastatic lymph nodes and treatment with LEV as significant prognostic factors for DFS. Therapy with IFN, grading and tumour localisation had no significant impact on DFS in the multiple analysis.
With regard to OS, sex, tumour size, the number of metastatic lymph nodes and again treatment with LEV were found to be significant prognostic variables. Therapy with IFN, grading, tumour localisation and age demonstrated no significant impact on OS in the Cox model. The results of the multiple analyses are shown in Tables 2 and 3.

An additional Cox analysis including the cumulatively delivered 5-FU dose per patient demonstrated this parameter to have a statistically significant impact on OS $(P<0.0001)$, but no significant influence on DFS $(P=0.1232)$. Adjusting for the cumulative 5-FU dose in the multiple analyses for DFS, the effect of IFN remained nonsignificant and the effect of LEV also remained approximately the same. Analogous multiple analyses for OS revealed that the effect of IFN was significant (HR 0.730, $P=0.0347$ ). The effect of LEV was slightly weaker (HR 1.334, $P=0.0418$ ) when adjusted for the cumulative 5-FU dose but remained statistically significant (all patients were considered in these analyses according to the intention-to-treat principle). When considering only patients who received at least one therapy cycle, three patients were excluded from the analyses and the effect of LEV on OS again remained significant. 


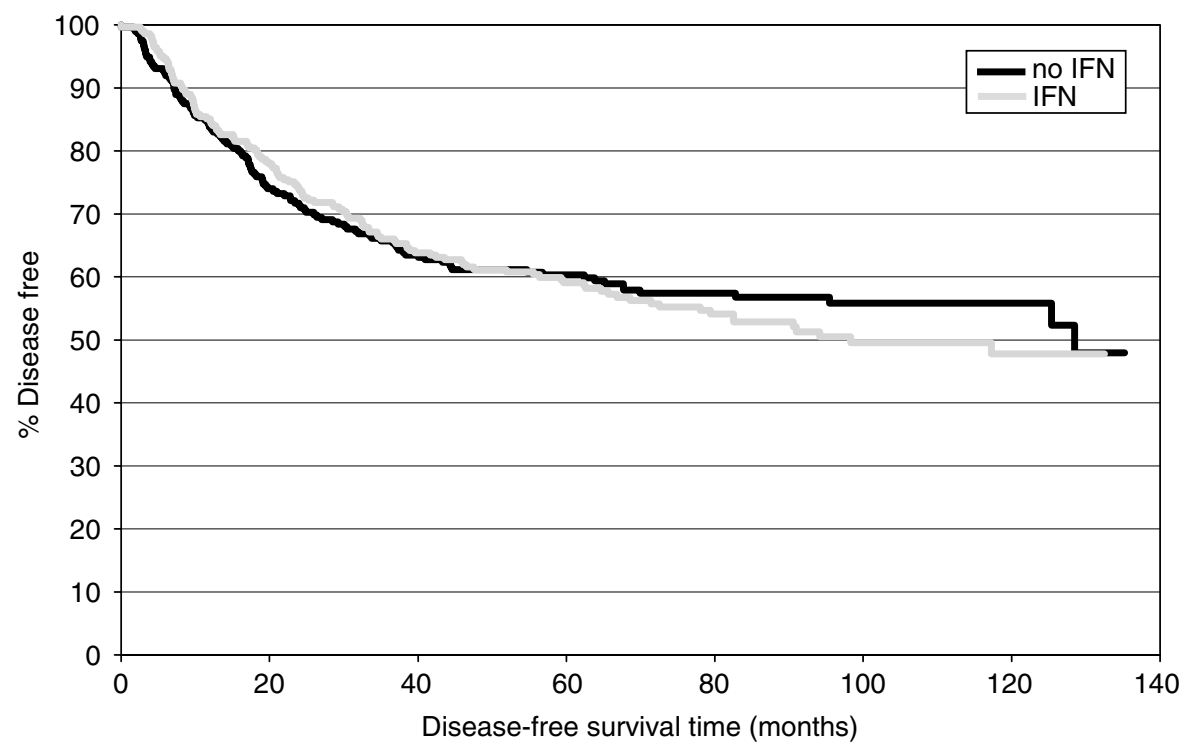

Figure 3 Disease-free survival: IFN vs no IFN.

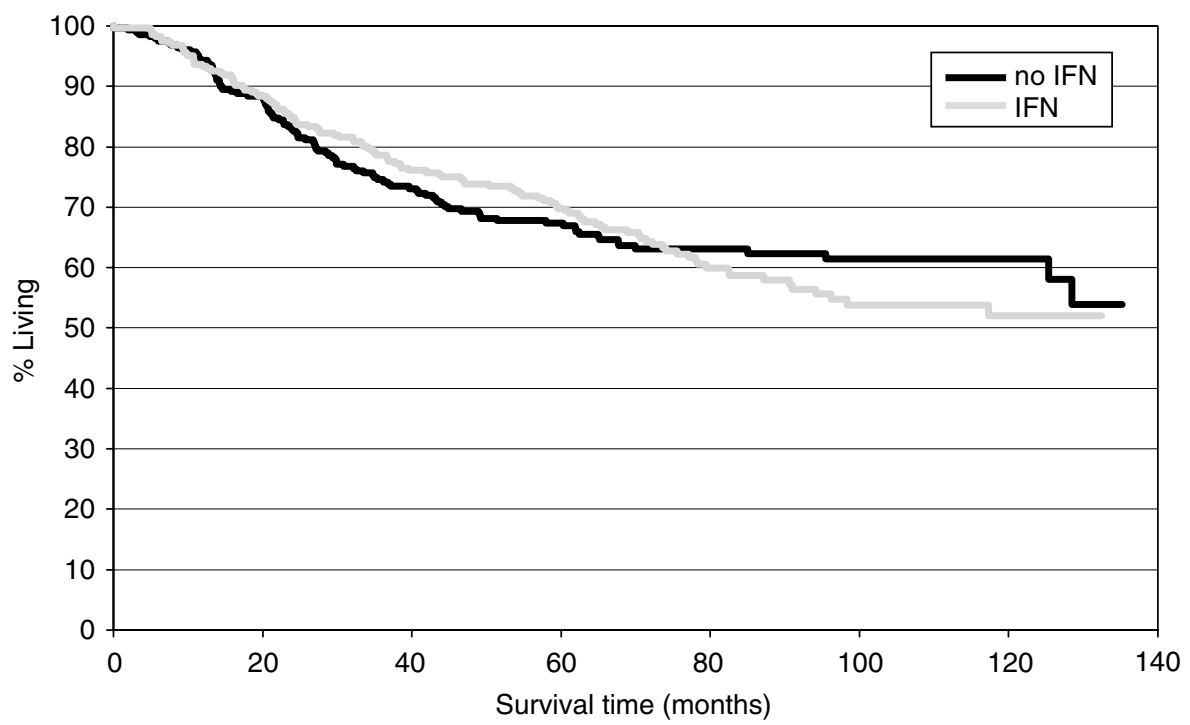

Figure 4 Overall survival: IFN vs no IFN.

Analysing interaction terms with respect to time, the multiple model revealed treatment with IFN as a time-dependent prognostic factor for DFS and OS, indicating that therapy with IFN reduces the risk of relapse and the risk of death temporarily, but not over the entire follow-up period (see Figures 3 and 4).

\section{Toxicity}

Grade 3 and 4 toxicities were documented during adjuvant therapy in $11.2 \%$ of the patients in the 5 -FU single-agent arm, in $21.5 \%$ of the patients treated with $5-\mathrm{FU} / \mathrm{LEV}$, in $26.7 \%$ of the patients in the 5 -FU/IFN arm and in $40.4 \%$ of the patients treated in the 5-FU/ LEV/IFN arm.

The distribution of grade 3 and 4 toxic effects is shown in Table 4.

\section{DISCUSSION}

At the time of initiation of this trial, the combination of 5-FU with LEV was considered as the standard adjuvant treatment of stage III colon cancer. However, in the final phase of recruitment to this trial, data from other studies showed that neither LEV nor IFN is of benefit in the adjuvant treatment of colon cancer. Final analysis data of the Intergroup trial INT 0089 suggested 5-FU plus leucovorin for 6 months to be preferable over 12 months of 5FU plus LEV for reasons of toxicity, costs and patients convenience (Haller et al, 1998). The NSABP trial C-04 compared the efficacy of 5-FU plus leucovorin with 5-FU plus LEV and with the triple combination of 5-FU plus leucovorin plus LEV. The study demonstrated a statistically significant prolongation of DFS in favour of 5-FU plus leucovorin compared to 5-FU plus LEV, but no significant difference between these two treatment arms in terms 
Table 2 Multiple analysis: evaluation of prognostic factors for DFS

\begin{tabular}{lccc}
\hline & HR & $\mathbf{9 5 \%} \mathbf{~ C l}$ & P-value \\
\hline Age & 1.134 & $1.011-1.273$ & 0.0325 \\
Sex & 1.331 & $1.036-1.711$ & 0.0255 \\
Tumour size & 1.432 & $1.119-1.831$ & 0.0043 \\
Number of metastatic lymph nodes & 1.369 & $1.058-1.77 \mid$ & 0.0169 \\
Histopathological grading & 1.241 & $0.950-1.621$ & 0.1132 \\
& & & \\
Location of tumour & & & 0.8169 \\
Left colon and sigmoid & 1.095 & $0.814-1.472$ & \\
Flexures and transverse colon & 1.097 & $0.766-1.571$ & \\
Treatment with LEV & 1.430 & $1.115-1.835$ & 0.0049 \\
Treatment with IFN & 0.942 & $0.736-1.206$ & 0.6364 \\
\hline
\end{tabular}

For the HR, the reference category for categorical covariates was female for sex, $\leqslant 3$ metastatic lymph nodes for number of metastatic lymph nodes, GI/G2 for histopathological grading, caecum and right colon for location of tumour, no LEV for treatment with LEV and no IFN for treatment with IFN. DFS= disease-free survival; LEV = levamisole; IFN = interferon alfa.

Table 3 Multiple analysis: evaluation of prognostic factors for OS

\begin{tabular}{lccc}
\hline & HR & $\mathbf{9 5 \%} \mathbf{~ C l}$ & P-value \\
\hline Age & 1.011 & $0.998-1.025$ & 0.0923 \\
Sex & 1.452 & $1.103-1.912$ & 0.0078 \\
Tumour size & 1.659 & $1.266-2.174$ & 0.0002 \\
Number of metastatic lymph nodes & 1.417 & $1.069-1.879$ & 0.0155 \\
Histopathological grading & 1.232 & $0.920-1.650$ & 0.1623 \\
& & & \\
Location of tumour & & & 0.7833 \\
Left colon and sigmoid & 1.121 & $0.812-1.548$ & \\
Flexures and transverse colon & 1.065 & $0.715-1.585$ & \\
Treatment with LEV & 1.460 & $1.110-1.920$ & 0.0069 \\
Treatment with IFN & 0.975 & $0.744-1.278$ & 0.8539 \\
\hline
\end{tabular}

For the HR, the reference category for categorical covariates was female for sex, $\leqslant 3$ metastatic lymph nodes for number of metastatic lymph nodes, GI/G2 for histopathological grading, caecum and right colon for location of tumour, no LEV for treatment with LEV and no IFN for treatment with IFN. OS=overall survival; $\mathrm{LEV}=$ levamisole; IFN = interferon alfa.

Table 4 Patients with grade 3 and 4 toxicities in the four treatment arms

\begin{tabular}{lccccr}
\hline & $\begin{array}{c}\text { 5-FU } \\
\text { (\%) }\end{array}$ & $\begin{array}{c}\text { 5-FU+ } \\
\text { LEV (\%) }\end{array}$ & $\begin{array}{c}\text { 5-FU+ } \\
\text { IFN (\%) }\end{array}$ & $\begin{array}{c}\text { 5-FU+LEV+ } \\
\text { IFN (\%) }\end{array}$ & P-value \\
\hline Nausea & 3.5 & 6.5 & 3.6 & 8.2 & 0.2197 \\
Diarrhoea & 3.5 & 3.6 & 2.9 & 3.4 & 0.9885 \\
Stomatitis & 0 & 2.2 & 0.7 & 1.4 & 0.2870 \\
Fever & 0 & 0.7 & 2.2 & 4.1 & 0.0331 \\
Leucopenia & 0.7 & 1.4 & 3.6 & 11.6 & $<0.0001$ \\
Thrombopenia & 0.7 & 1.4 & 2.2 & 1.4 & 0.8806 \\
Alopecia & 0 & 0.7 & 0.7 & 1.4 & 0.7606 \\
Other toxic effects & 2.8 & 5.0 & 10.8 & 8.9 & 0.0488 \\
\hline
\end{tabular}

5-FU = 5-fluorouracil; LEV = levamisole; IFN = interferon alfa.

of OS. The comparison between patients treated with 5-FU plus leucovorin and those who received 5-FU plus leucovorin plus LEV showed no difference for either DFS or OS (Wolmark et al, 1999). The NSABP protocol C-05 evaluated the efficacy of the addition of interferon alfa-2a to 5-FU and leucovorin in the adjuvant treatment of colon cancer. The results of this trial showed no significant difference either in DFS or in OS for the IFN arm (Wolmark et al, 1998). Owing to the absence of significant differences in OS in these trials examining the role of LEV and IFN in the adjuvant setting of colon cancer, continuation of accrual in the present trial was considered as ethically justifiable until the end of the accrual period in 1999.

In this trial, it was documented for the first time that the combination of LEV with 5-FU as adjuvant treatment of stage III colon cancer is significantly inferior in terms of DFS and OS compared to a therapy with 5-FU alone. Owing to the finding that patients in the LEV-containing therapy arms received less cumulative 5-FU, the unfavourable effect of LEV could be assumed to be caused by the lower 5-FU dose only. However, the multiple analysis adjusting for differences in the cumulatively delivered 5FU dose in the treatment groups caused only a minor change of observed hazard ratio for the therapy effect of LEV. Thus, it can be ruled out that the observed differences in DFS and OS are exclusively a consequence of different 5 -FU doses in the treatment arms. Levamisole affects survival independently of the delivered cumulative 5-FU dose. These findings contribute to assess the efficacy of LEV in 5-FU-based adjuvant therapy, since the Intergroup trial published by Moertel et al (1990), which led to the NIH consensus suggesting 5-FU and LEV as standard treatment for stage III colon cancer, compared 5-FU plus LEV with observation and with LEV alone. The Intergroup trial revealed, for the single-agent LEV arm, an identical OS to no adjuvant therapy. Another study addressing the role of LEV in colon cancer, the Quick and Simple and Reliable (QUASAR) trial, investigated a possible survival benefit by using a higher dose of folinic acid or by addition of LEV to 5-FU and folinic acid and found no survival benefit from LEV (QUASAR Collaborative Group, 2000). Patients treated with LEV had an even higher, although statistically not significant, odds ratio for disease recurrence and death compared to patients without LEV therapy. The observed trend to deterioration of prognosis by addition of LEV in the QUASAR trial is not concordant with the findings of our study, which shows a significantly detrimental effect of LEV in 5-FU-based adjuvant therapy of colon cancer. A possible explanation for the more distinct survival disadvantage for the LEV arm in the present study could be the higher 5-FU dose density in a fraction of the QUASAR study population, whereas the dose density of LEV was the same as in the present study.

In view of the findings of the present study, an antagonising effect of LEV on 5-FU can be suggested. Furthermore, the findings of this trial correlate very well with the results of a recently published preclinical study, which found that LEV did not only lack a significant growth inhibitory effect as a single agent in colon cancer cell lines, but also antagonised 5-FU-induced tumour growth inhibition when combined with this cytostatic agent (Wiebke et al, 2003).

The results of the present study imply that the addition of LEV to 5-FU in the adjuvant treatment of colon cancer has an unfavourable effect on patient prognosis and should therefore not be considered as a possible adjuvant treatment option.

A smaller trial comparing an adjuvant therapy with 5-FU plus LEV for 1 year with 5-FU plus folinic acid, administered for either 6 or 12 months, showed that 6 or 12 months of treatment with 5FU plus folinic acid is equivalent to 12 months with 5-FU plus LEV (Dencausse et al, 2002).

Another study comparing 5-FU plus leucovorin with 5-FU plus LEV, both for 1 year as adjuvant therapy in patients with resected stage III colon cancer, showed a significant superiority of the 5-FU plus leucovorin arm in terms of DFS and OS (Arkenau et al, 2003).

The INTACC trial investigating whether the addition of leucovorin to the combination of 5-FU plus LEV improves survival in patients with Dukes' B and C colon cancer showed no statistical difference in DFS and OS between the 5-FU/LEV and the 5-FU/ LEV/leucovorin treatment arms (Di Costanzo et al, 2003).

The second goal of the present study was to examine the effect of IFN in a 5-FU-based adjuvant therapy for stage III colon cancer. 
Although Kaplan-Meier curves suggest a survival advantage within the first years of follow-up for patients treated with IFN, this early benefit in terms of DFS and OS is counterbalanced by a higher number of disease relapses and deaths at later periods of follow-up. Higher toxicity and a higher frequency of premature therapy cessations resulted in a significantly lower cumulative 5FU delivery in the IFN treatment arms, which could be a reason for the missing effect of IFN on DFS and OS. In fact, the multiple model adjusting for the delivered 5-FU dose demonstrates a significantly positive impact of IFN on OS, but not on DFS. As 5FU dose is different between therapy groups, it cannot be ruled out that patients receiving a higher 5-FU dose are a selected group with good prognostic factors. Thus, the improved OS hazard ratio for the IFN treatment group in the 5-FU adjusted model could be

\section{REFERENCES}

Arkenau HT, Bermann A, Rettig K, Strohmeyer G, Porschen R, on behalf of the Arbeitsgemeinschaft Gastrointestinale Onkologie (2003) 5-Fluorouracil plus leucovorin is an effective adjuvant chemotherapy in curatively resected stage III colon cancer: long-term follow-up results of the adjCCA-01 trial. Ann Oncol 14: 395-399

Arnaud JP, Buyse M, Nordlinger B, Martin F, Pector JC, Zeitoun P, Adloff A, Duez N (1989) Adjuvant therapy of poor prognosis colon cancer with levamisole: results of an EORTC double-blind randomized clinical trial. Br J Surg 76: 284-289

Buroker TR, Moertel CG, Fleming TR, Everson LK, Cullinan SA, Krook JE, Mailliard JA, Marschke RF, Klaassen DJ, Laurie JA, Moon MD (1985) A controlled evaluation of recent approaches to biochemical modulation or enhancement of 5-fluorouracil therapy in colorectal carcinoma. J Clin Oncol 3: $1624-1631$

Cox DR (1972) Regression models and life-tables. J R Stat Soc [B] 34: $187-220$

Dencausse Y, Hartung G, Sturm J, Kopp-Schneider A, Hagmuller E, Wojatschek C, Lindemann H, Fritze D, Queisser W (2002) Adjuvant chemotherapy in stage III colon cancer with 5-fluorouracil and levamisole versus 5-fluorouracil and leucovorin. Onkologie 25: 426-430

Di Costanzo F, Sobrero A, Gasperoni S, Dogliotti L, Frassineti L, Falcone A Lionetto R, Bruzzi P, Luppi G, Gallo L, Conte P, Comandone A, Turci D, Marzola M, Folco U, Pfanner E, Mestriner M, Boni C, Galli C, Tonato M, Rosso R, on behalf of the Italian National Intergroup of Adjuvant Chemotherapy in Colon Cancer (INTACC (2003) Adjuvant chemotherapy in the treatment of colon cancer: randomized multicenter trial of the Italian National Intergroup of Adjuvant Chemotherapy in Colon Cancer (INTACC). Ann Oncol 14: 1365 - 1372

Haller DG, Catalano PJ, Mac Donald JS (1998) Fluorouracil (FU), leucovorin (LV) and levamisole (LEV) adjuvant therapy for colon cancer: five-year final report of INT-0089. Proc Am Soc Clin Oncol 17: 256 (abstract 982)

Kaplan EL, Meier P (1958) Nonparametric estimation from incomplete observations. J Am Stat Assoc 53: 457-481

Laurie JA, Moertel CG, Fleming TR, Wieand HS, Leigh JE, Rubin J, McCormack GW, Gerstner JB, Krook JE, Malliard J, Twito DI, Morton RF, Tschetter LK, Barlow JF (1989) Surgical adjuvant therapy of largebowel carcinoma: an evaluation of levamisole and the combination of levamisole and fluorouracil - The North Central Cancer Treatment Group and the Mayo Clinic. J Clin Oncol 7: 1447-1456

Mantel N (1966) Evaluation of survival data and two new rank order statistics arising in its consideration. Cancer Chemother Rep 50: $163-170$ Moertel CG, Fleming TR, Macdonald JS, Haller DG, Laurie JA, Goodman PJ, Ungerleider JS, Emerson WA, Tormey DC, Glick JH, Veeder MH, Maillard JA (1990) Levamisole and fluorouracil for adjuvant therapy of resected colon carcinoma. $N$ Engl J Med 322: 352-358

\section{Appendix A}

Apart from the authors of this article, members of the Austrian Breast and Colorectal Cancer Study Group participating in this trial included the following: E Andritsch, $\mathrm{H}$ Bacher, T Bauernhofer, A Berger, O Böckl, K Czerwenka, D Depisch, W Draxler, P Dubsky, A El-Shabrawi, J Freisinger, B Gebhard, P Götzinger, explained by age or other unobserved nontreatment-related prognostic factors also. These findings confirm the results of other trials that the addition of IFN to 5-FU does not confer a survival benefit (Wolmark et al, 1998).

Frequency of grade 3 and 4 toxicities was increased in therapy arms including LEV or IFN. The highest rate of severe toxicities was documented within the patient group treated with the tripleagent combination. Premature discontinuation of therapy was most frequent in the treatment arms containing IFN; the highest number of therapy cessations was observed in the 5-FU/LEV/IFN arm.

Summarising the results of this study, neither LEV nor IFN showed efficacy in a 5-FU-based adjuvant therapy for stage III colon cancer.

NIH consensus conference (1990) Adjuvant therapy for patients with colon and rectal cancer. JAMA 264: 1444-1450

Parkin DM, Bray FI, Devesa SS (2001) Cancer burden in the year 2000. The global picture. Eur J Cancer 37: S4-S66

Pocock SJ, Simon R (1975) Sequential treatment assignment with balancing for prognostic factors in the controlled clinical trial. Biometrics 31: $103-115$

QUASAR Collaborative Group (2000) Comparison of flourouracil with additional levamisole, higher-dose folinic acid, or both, as adjuvant chemotherapy for colorectal cancer: a randomised trial. Lancet 355: $1588-1596$

Sertoli MR, Guarneri D, Rubagotti A, Porcile G, Nobile MT, Rosso R (1987) Adjuvant immunochemotherapy in colorectal cancer Dukes C. Oncology 44: $78-81$

Wadler S, Lembersky B, Atkins M, Kirkwood J, Petrelli N (1991) Phase II trial of fluorouracil and recombinant interferon alfa-2a in patients with advanced colorectal carcinoma: an Eastern Cooperative Oncology Group study. J Clin Oncol 9: 1806-1810

Wadler S, Schwartz EL, Goldman M, Lyver A, Rader M, Zimmerman M, Itri L, Weinberg V, Wiernik PH (1989) Fluorouracil and recombinant alfa2a-interferon: an active regimen against advanced colorectal carcinoma. J Clin Oncol 7: 1769-1775

Wadler S, Wersto R, Weinberg V, Thompson D, Schwartz EL (1990) Interaction of fluorouracil and interferon in human colon cancer cell lines: cytotoxic and cytokinetic effects. Cancer Res 50: 5735-5739

Wiebke EA, Grieshop NA, Loehrer PJ, Eckert GJ, Sidner RA (2003) Antitumor effects of 5-fluorouracil on human colon cancer cell lines: antagonism by levamisole. J Surg Res 111: 63-69

Wolmark N, Bryant J, Smith R, Grem J, Allegra C, Hyams D, Atkins J, Dimitrov N, Oishi R, Prager D, Fehrenbacher L, Romond E, Colangelo L, Fisher B (1998) Adjuvant 5-fluorouracil and leucovorin with or without interferon alfa-2a in colon carcinoma: National Surgical Adjuvant Breast and Bowel Project protocol C-05. J Natl Cancer Inst 90: 18101816

Wolmark N, Fisher B, Rockette H, Redmond C, Wickerham DL, Fisher ER, Jones J, Glass A, Lerner H, Lawrence W, Prager D, Wexler M, Evans J, Cruz A, Dimitrov N, Jochimsen P (1988) Postoperative adjuvant chemotherapy or BCG for colon cancer: results from NSABP protocol C-01. I Natl Cancer Inst 80: 30-36

Wolmark N, Rockette H, Mamounas E, Jones J, Wieand S, Wickerham DL, Bear HD, Atkins JN, Dimitrov NV, Glass AG, Fisher ER, Fisher B (1999) Clinical trial to assess the relative efficacy of fluorouracil and leucovorin, fluorouracil and levamisole, and fluorouracil, leucovorin, and levamisole in patients with Dukes' B and C carcinoma of the colon: results from National Surgical Adjuvant Breast and Bowel Project C-04. J Clin Oncol 17: $3553-3559$

T Grünberger, K Haider, M Hanak, B Hartmann, H Hauser, J Hebenstreit, E Hell, G Hofmann, D Hussian, G Jatzko, C Kainz, D Kandioler, A Kasparek, R Kolb, P Konstantiniuk, G Kosina, P Krippl, L Kronberger, I Kuss, W Kwasny, M Lang, G Locker, H Ludwig, H Luschnik, K Mach, P Mayer, R Menzel, G Michlmayr, 
E Moritz, A Obermair, D Nitsche, I Papadi, C Papp, J Pfeifer, F Ploner, U Pluschnig, R Pointner, C Rass, A Reiner, G Reiner, S Reinisch, M Riegler, S Roka, G Rosanelli, M Rudas, G Russ, H Samonigg, R Schandalik, M Schemper, M Schmid, W Schwaiger, U

Sevelda, M Smola, H Spoula, P Steindorfer, H Stöger, S Taucher, C Tausch, J Tschmelitsch, G Tschurtschenthaler, M Umlauft, N Vavra, P Wagner, G Wahl, M Wehrschütz, C Wenzel and C Zielinski. 